\title{
Serum Thrombomodulin to Predict Severity in Pediatric Sepsis-Fancy or Frenzy?
}

\author{
Namita Ravikumar ${ }^{1}(1) \cdot$ Arun Bansal $^{2}$ (1)
}

Received: 27 May 2021 / Accepted: 3 June 2021/Published online: 24 June 2021

(c) Dr. K C Chaudhuri Foundation 2021

Sepsis is a state of dysregulated host immune response characterized by inflammation, endothelial dysfunction, and microcirculatory derangements leading to organ dysfunction [1]. Inflammatory cytokines, markers of endothelial dysfunction, and coagulation pathway molecules have been utilized to assess the severity of sepsis [2]. Thrombomodulin (TM) is an endothelial thrombin receptor that inhibits the procoagulant action of thrombin and enhances the anticoagulant and anti-inflammatory properties of protein C. Soluble form of TM is released into the circulation during endothelial cell damage [3]. High serum TM in acute respiratory distress syndrome, disseminated intravascular coagulation (DIC), and sepsis-induced organ dysfunction reflect endothelial injury as well as disease activity in vasculitis [4-6].

Khattab et al. found in their study published in this issue, that serum TM levels were significantly elevated in children with systemic inflammatory response syndrome (SIRS) $(2.88 \pm 0.17)$, sepsis $(3.94 \pm 0.80)$, and severe sepsis $(5.49 \pm 0.22)$, showing progressively increasing values with severity in comparison with controls $(2.34 \pm 0.17)$ [7]. Mechanically ventilated children and nonsurvivors had significantly higher TM levels. A cutoff value $>3.5 \mathrm{mU} / \mathrm{mL}$ had higher sensitivity $(84 \%)$ and specificity $(100 \%)$ than $\mathrm{C}$-reactive protein and white blood cell count for prediction of sepsis (AUC 0.915, 0.789, and 0.709, respectively). Serum TM levels also correlated positively with the severity of illness scores like Pediatric Risk of Mortality-III and Pediatric Index of Mortality -2. However, for mortality prediction, these scores fared better than serum TM levels. This study highlights the utility of serum TM (measured within

Arun Bansal

drarunbansal@gmail.com

1 Department of Pediatrics, All India Institute of Medical Education and Research, New Delhi, India

2 Department of Pediatrics, Advanced Pediatrics Centre, Postgraduate Institute of Medical Education and Research (PGIMER), Chandigarh, India
$24 \mathrm{~h}$ of admission) as a prognostic marker and predictor of severity in pediatric sepsis.

Timely prediction of severity gives an edge to manage sepsis and prevent progressive organ dysfunction. It also opens up avenues for newer therapeutic modalities targeting biological pathways of sepsis. The recombinant human soluble TM has been tried for sepsis-associated coagulopathy in the SCARLET trial but failed to show mortality benefit [8]. Inflammatory cytokines and coagulation pathway markers haveshown correlation with sepsis severity and predict DIC early in sepsis, respectively [9, 10]. Pediatric Sepsis Biomarker Risk Model (PERSEVERE), a risk-stratification tool incorporating five biomarkers, developed and validated by Wong et al. to predict pediatric septic shock mortality, has shown a sensitivity of $93 \%$, specificity $74 \%$, positive predictive value $32 \%$, and negative predictive value of $99 \%$ [11].

The sepsis-3 definition has done away with the terms SIRS and severe sepsis and mandated the need for organ dysfunction to define sepsis based on Sequential Organ Failure Assessment (SOFA) score and bedside quick-SOFA (qSOFA) to identify high-risk patients [12]. Clinical definitions applied at the bedside to detect progressive organ dysfunction are likely to be more useful in the titration of management than most fancy expensive biomarker tools that have a long turnaround time and are best suited for research purposes. Sepsis demonstrates a marked heterogeneity in clinical phenotype among individuals, and no single biomarker would be able to accurately diagnose sepsis, monitor response, or predict severity. Each of the biomarkers or combinations may be used judiciously to understand sepsis-related inflammatory pathobiology phenotypes that may have therapeutic implications [13].

\section{Declarations}

Conflict of Interest None. 


\section{References}

1. Martin K, Weiss SL. Initial resuscitation and management of pediatric septic shock. Minerva Pediatr. 2015;67:141-58.

2. Carlton EF, McHugh WM, McDonough K, Sturza J, Desch K, Cornell TT. Markers of endothelial dysfunction and cytokines in high-risk pediatric patients with severe sepsis. Am J Respir Crit Care Med. 2020;201:380-4.

3. Boffa MC, Karmochkine M. Thrombomodulin: an overview and potential implications in vascular disorders. Lupus. 1998; 7:s120-5.

4. Sapru A, Calfee CS, Liu KD, et al. Plasma soluble thrombomodulin levels are associated with mortality in the acute respiratory distress syndrome. Intensive Care Med. 2015;41:470-8.

5. Lin JJ, Hsiao HJ, Chan OW, Wang Y, Hsia SH, Chiu CH. Increased serum thrombomodulin level is associated with disease severity and mortality in pediatric sepsis. PLoS One. 2017;12:1-10.

6. Boehme MWJ, Schmitt WH, Youinou P, Stremmel WR, Gross WL. Clinical relevance of elevated serum thrombomodulin and soluble E-selectin in patients with Wegener's granulomatosis and other systemic vasculitides. Am J Med. 1996;101:387-94.

7. Khattab AA, Dawood AAER, Saleh NY. Value of thrombomodulin as a marker for sepsis in critically ill children. Indian J Pediatr. 2020. https://doi.org/10.1007/s12098-020-03564-w.
8. Vincent JL, Francois B, Zabolotskikh I, et al. Effect of a recombinant human soluble thrombomodulin on mortality in patients with sepsis-associated coagulopathy: the SCARLET randomized clinical trial. JAMA. 2019;321:1993-2002.

9. Angurana SK, Bansal A, Muralidharan J, Aggarwal R, Singhi S. Cytokine levels in critically ill children with severe sepsis and their relation with the severity of illness and mortality. J Intensive Care Med. 2021;36:576-83.

10. Masuda T, Shoko T, Deguchi Y. Clinical investigation of coagulation markers for early detection of sepsis-induced disseminated intravascular coagulation: a single-center, prospective observational study. Clin Appl Thromb Hemost. 2018;24:1082-7.

11. Wong HR, Salisbury S, Xiao Q, et al. The pediatric sepsis biomarker risk model. Crit Care. 2012;16:R174.

12. Singer M, Deutschman CS, Seymour CW, et al. The third international consensus definitions for sepsis and septic shock (Sepsis-3). JAMA. 2016;315:801-10.

13. Carcillo JA, Halstead ES, Hall MW, et al. Three hypothetical inflammation pathobiology phenotypes and pediatric sepsisinduced multiple organ failure outcome. Pediatr Crit Care Med. 2017;18:513-23.

Publisher's Note Springer Nature remains neutral with regard to jurisdictional claims in published maps and institutional affiliations. 